Agosto 2012 | www.ocula.it - DOI: 10.12977/ocula11

\title{
Politiche della sicurezza e forme del controllo Una prospettiva semiotica
}

\author{
di Pierluigi Cervelli \\ Università di Roma La Sapienza-pierluigi.cervelli@gmail.com
}

\begin{abstract}
Security policies and forms of control

A semiotic perspective

The aim of this article is to reflect on the political control of urban space and population mobility, particularly in relation to government measures and regulations governing the management of the hosting camps of the Rom and Sinti within the Municipality of Rome (the so-called "solidarity villages"). To this end, the articledevelops as a discourse analysis of the number of documents and regulations passed by the Berlusconi government, part of which wasrejected by the Italian Council of State (November 2011). The thesis of the article is that the new regulations of Rom camps are designed to control the movement of Rom and Sinti, who live in them, however, with the explicit aim of preventing permanent accommodation to people housed in them. From this point of view, the article argues that the measures analyzed create nomadism who declare to be reason to their own needs. The conclusions of the work finally supports the actuality of the reflections on security proposed by Michel Foucault (2004), showing how they can be applied to new fields of research interacting with the concepts and methods of semiotic analysis.
\end{abstract}

\section{Keywords}

Semiotics, Political discourse, Rom and Sinti, Urban space, Security

\section{Sommario}

1. Lo spazio dell'emergenza

2. Dall'indeterminazione alla paura

3. Forme del controllo: spazio visibile e confini mobili

4. Conclusioni

Bibliografia 


\section{Ocula ${ }^{13}$}

Architettura e politica: un incrocio di sguardi | Pierluigi Cervelli, Politiche della sicurezza e forme del controllo. Una prospettiva semiotica.

Un tema che è insieme sconfinato e puntuale, quello del rapporto fra architettura e politica, richiede la precisazione preliminare del punto di vista che si intende assumere, e con esso della scala di analisi che si intende adottare. Il mio obiettivo sarà quello di considerare l'architettura del territorio su una scala urbana, e di fare qualche riflessione sul modo in cui sono definiti i regimi di mobilità della popolazione: la sua concentrazione $\mathrm{e}$ la sua dispersione, le temporalità che questi regimi definiscono. L'ipotesi alla base di questa impostazione è che questa definizione abbia un valore politico, contribuendo a determinare (definendo luoghi modalizzati, del "vietato", dell' "obbligatorio", del "possibile") il campo delle relazioni sociali fra collettività culturali che coabitano e le loro forme di subordinazione ad una autorità generale ${ }^{1}$, ponendo, in termini più ampi, il problema della gestione spaziale e politica della diversità culturale e sociale.

Il corpus analitico sarà costituito da una serie di ordinanze e provvedimenti amministrativi, emessi fra il 2007 ed il 2009 dal governo italiano e dai sindaci di Roma e Milano, in relazione alla presenza di comunità rom ${ }^{2}$ nei territori comunali. Si tratta dell'Ordinanza del Presidente del Consiglio dei ministri del 30 Maggio 2008 "Disposizioni urgenti di protezione civile per fronteggiare lo stato di emergenza in relazione agli insediamenti di comunità nomadi nel territorio della regione Lazio (n. 3676)", delle "Linee guida per l'attuazione delle ordinanze del Presidente del Consiglio dei ministri del 30 maggio 2008, $\mathrm{n}^{\circ} 3676,3677,3678$, concernenti insediamenti di comunità nomadi nelle regioni Campania, Lazio e Lombardia", dei "Patti per Roma sicura" del 2007 e del 2008 firmati dai sindaci della città (2007, W. Veltroni; 2008, G. Alemanno), dal prefetto, dai presidenti della Regione Lazio e della Provincia di Roma, alla presenza del ministro degli Interni; e infine del "Regolamenti delle aree destinate ai nomadi" del Comune di Milano e del "Regolamento per la gestione dei villaggi attrezzati per le comunità nomadi nella regione Lazio" emessi rispettivamente dal Commissario per l'emergenza nomadi in Lombardia e dal Commissario Delegato per l'emergenza nomadi nella regione Lazio3. Di

${ }^{1}$ Il presupposto di una simile impostazione è che l'architettura parli d'altro che di sé stessa (Hammad 2003; Greimas 1976) e che non lo faccia come un sistema simbolico, un sistema cioè in cui la relazione fra elementi espressivi e di contenuto sia non commutabile e conforme: immobile e biunivoca. Uno degli obiettivi di questa analisi sarà invece proprio mostrare come lo spazio urbano possa funzionare localmente come una semiotica procedendo analiticamente per correlazioni fra complessi strutturali dell'espressione e del contenuto. Rispetto all'accezione semiotica di "politica" mi sembra pertinente con l'approccio di questo saggio quella fornita da E. Landowski (1989: 275): «origine di una dimensione politica ogni "fare" discorsivo il cui svolgimento mira, o semplicemente comporta, qualche effetto di potere, intendendo con questo la trasformazione delle competenze modali delle parti partecipanti alla comunicazione e, in seguito, la trasformazione delle condizioni di realizzazione dei loro programmi rispettivi di azione». Sullo stesso tema cfr. anche Fabbri e Marcarino (1985) e Montanari (2010).

2 Uso il termine rom per esigenze di "economia", indicando con esso sia rom che sinti (ed in realtà le molte e diverse comunità che si definiscono rom o sinti), e più in generale quei cittadini che nelle ordinanze citate saranno definiti "nomadi".

3 Tutti i documenti sono reperibili sul sito del Ministero degli Interni italiano (www.interno.it).

2 | www.ocula.it | agosto 2012 


\section{Ocula ${ }^{13}$}

Architettura e politica: un incrocio di sguardi | Pierluigi Cervelli, Politiche della sicurezza e forme del controllo. Una prospettiva semiotica.

questi documenti considererò solo gli aspetti pertinenti per il tema oggetto di questo lavoro.

Il paragrafo 1 è dedicato alla presentazione della situazione in cui è stata dichiarata l'emergenza. Il paragrafo 2 analizza le modalità discorsive attraverso cui i provvedimenti lasciano indefinite le componenti spaziali temporali e attoriali dell'emergenza. Il paragrafo 3 è infine dedicato all'articolazione dei nuovi spazi in cui ospitare i rom e al controllo dell'accessibilità allo spazio urbano previsto in questi nuovi campi sosta.

\section{Lo spazio dell'emergenza}

I provvedimenti che consideriamo sono stati emessi fra il 2007 ed il 2009. In quello stesso periodo il governo italiano ha dichiarato lo stato di emergenza nazionale per l'afflusso degli immigrati e, nella città di Roma si è "improvvisamente" scoperto che esistevano più di cento insediamenti irregolari abitati da rom, pricipalmente provenienti dalla ex-Jugoslavia e dalla Romania 4 . Appena eletto, l'attuale sindaco di Roma Giovanni Alemanno ha affermato che era necessario espellere 20.000 rom dal territorio comunale. Sulla base dei dati forniti dall'Arci Karin, desunti da un censimento aggiornato all'agosto del 2008, ho potuto quantificare le presenze, trovando che erano poco più di $12.000^{5}$. Quello che mi è parso però più interessante era la disposizione dei campi (in particolare di quelli non autorizzati): è stato possibile infatti dimostrare come dopo l'inizio degli sgomberi, nel 2007 (durante l'amministrazione del sindaco W. Veltroni), il numero dei campi abusivi fosse raddoppiato rispetto alle rilevazioni del 2006 e le loro dimensioni si fossero drasticamente ridotte. Estremamente piccoli e distribuiti in tutta la città (in 18 municipi su 19, compresa l'area del centro storico della città), i campi evidenziavano un'interpretazione strategica dello spazio urbano, basata sull'inserimento interstiziale nelle parti invisibili della città (nei sottopassaggi abbandonati, nel cuore inaccessibile dei parchi o all'interno delle reti infrastrutturali; sotto i ponti autostradali, le tangenziali e le ferrovie) e su un modello di spazio urbano tridimensionale e stratificato, riferibile a categorie semantiche come visibile/invisibile, che si opponeva radicalmente alla categorizzazione dello spazio presupposta dalle forme di insediamento degli altri immigrati, basata su categorie come interno/esterno e dunque su opposizioni come centro/periferia. Questo non riconoscimento dei confini e delle gerarchie dello spazio urbano, riconducibile ad una distribuzione insediativa

\footnotetext{
4 L'allarme per la presenza di questi insediamenti, presenti fin dagli anni sessanta a Roma, si è acuito dopo l'omicidio, che ha avuto ampia risonanza mediatica, di una donna italiana, Giovanna Reggiani, da parte di un giovane rom, Romulus Nicolae Mailat, abitante di uno di questi campi e segnalato subito da altri rom alla polizia intervenuta per accertamenti.

5 Il dato è stato ottenuto sommando i 4179 abitanti dei campi irregolari ai 7900 (circa), che secondo i dati Caritas, abitavano nel 2006 nei campi "attrezzati o semiattrezzati” riconosciuti dal Comune di Roma. Il dato complessivo registra 12.079 persone.
} 


\section{Ocula ${ }^{13}$}

Architettura e politica: un incrocio di sguardi | Pierluigi Cervelli, Politiche della sicurezza e forme del controllo. Una prospettiva semiotica.

polverizzata e acentrica ${ }^{6}$, permetteva ai rom di essere dentro la città senza essere visibili e di potervi restare nonostante gli sgomberi continui. Questo spiegava inoltre, attraverso una comparazione fra la concentrazione della popolazione e la sua articolazione territoriale, come quella che sembrava una proliferazione di nuovi insediamenti era in realtà frutto di uno spostamento continuo e frammentario7.

I provvedimenti che considereremo fanno tutti riferimento all'emergenza legata al movimento di questa "popolazione senza territorio8", che sembra aver prodotto due tipi di risposte strategiche, entrambe politiche (in senso semiotico) in quanto negano questa forma insediativa: una discorsivolegislativa, legata in particolare ai provvedimenti per la sicurezza e ai "Patti per la sicurezza urbana", e una invece che potremmo definire "spaziale e abitativa" (in mancanza di termini migliori), relativa al modo in cui sono stati costruiti alcuni nuovi quartieri residenziali e alla articolazione e gestione dello spazio che potremmo definire "pubblico" al loro interno. Mi soffermerò sulla prima di queste risposte analizzando, principalmente dal punto di vista della sintassi discorsiva, i regolamenti e le normative relative ai nuovi campi sosta, cercando di evidenziare il modo in cui la loro articolazione semantica funzioni come una negazione strategica della modalità insediativa dei rom rilevata nel territorio del comune di Roma9.

\section{Dall'indeterminazione alla paura}

Il titolo del documento principale fra i provvedimenti è "Disposizioni urgenti di protezione civile per fronteggiare lo stato di emergenza in relazione agli insediamenti di comunità nomadi nel territorio della regione Lazio". Si tratta di una ordinanza emanata dal Primo ministro italiano nel 2008. La configurazione discorsiva che il titolo pone evidenzia immediatamente un'isotopia del conflitto che implica una configurazione narrativa polemica: due soggetti si fronteggiano, o meglio uno dei due si erge a contrastare il pericolo che l'altro rappresenta, evidente e incombente ma senza confini definiti. La natura del conflitto è infatti particolare: non dichiarato, improvviso, evidente e diffuso ${ }^{10}$.

${ }^{6}$ Cfr. Piasere 1999.

7 Cfr. Cervelli 2008.

8 Il "Patto per Roma sicura" del 2007 pone come suo obiettivo assicurare "interventi risolutivi delle esigenze di contenimento delle popolazioni senza territorio" (pag. 3).

9 Questo aspetto concerne il problema della traduzione (intersemiotica) fra forme discorsive e spaziali. In questo senso il riferimento è alla concezione della traduzione come metameccanismo di creazione di equivalenze (Greimas e Courtés, 1979-2007, voci "Traduzione" e "Parafrasi").

${ }^{10}$ Il tema dell'emergenza meriterebbe una riflessione ben più articolata e complessa. In questa sede ci siamo limitati ad un'analisi di tipo dizionariale, attraverso la considerazione di tre lessemi, "emergenza", "urgente" e "fronteggiare". Secondo il Grande dizionario Garzanti della lingua italiana (1987) l'“emergenza" è: 1) "l'emergere, ciò che emerge, che sporge" 2) "situazione particolarmente critica, difficile (...) stato di emergenza: situazione di pericolo grave e generalizzato su un certo territorio che impone alle autorità pubbliche di prendere particolari misure." Alla voce "emergere" il dizionario riporta invece la seguente definizione: 1) "affiorare 


\section{Ocula ${ }^{13}$}

Architettura e politica: un incrocio di sguardi | Pierluigi Cervelli, Politiche della sicurezza e forme del controllo. Una prospettiva semiotica.

Quello che mi pare più interessante è che la narrazione dell'emergenza si articola sulla base di alcuni elementi privi di determinazione:

1) un grande pericolo generalizzato su un territorio (come indicano le definizioni dizionariali "affiorato da un liquido": senza dunque delimitazioni spaziali precise)

2) un pericolo che è apparso con evidenza e velocità (senza indicazioni temporali che ne indichino però l'inizio)

3) un pericolo che preme, incalza e incombe

4) un pericolo cui si deve tenere testa al più presto, perché l'attacco è evidentemente in corso

Il testo non indica il crimine, né chi ne è l'autore, né contro chi è diretto o dove si effettua. Cos'è avvenuto dunque? Il nemico si è già dispiegato, e la sua modalità di posizionamento nel territorio è implicita nella configurazione discorsiva conflittuale che il titolo porta con sé. Questa indeterminatezza è coerente con quanto emergerà dall'analisi dell'intero documento.

Le motivazioni alla base dell'ordinanza del Presidente del Consiglio dei Ministri del 30 maggio 2008, che dichiara lo stato di emergenza, rafforzano questa indeterminatezza: "considerata la situazione di estrema criticità determinatasi nel territorio della regione Lazio, con particolare riferimento alle aree urbane del comune di Roma e alle zone circostanti, a causa della presenza di numerosi cittadini extracomunitari irregolari e nomadi che si sono stabilmente insediati nelle predette aree" (pag. 1). Notiamo prima di tutto come gli attori dell'emergenza siano tematizzati in maniera intimamente contraddittoria: "immigrati clandestini"1 che hanno manifestato una "presenza" e "nomadi"12 che "si sono insediati stabilmente"13.

da un liquido, venire in superficie" 2) "(estens.) venir fuori, mostrarsi al di sopra, apparire" 3) "(fig.) risultare, manifestarsi con chiarezza, con evidenza".

L'etimologia latina, da ex-mergere, ossia letteralmente "tuffare fuori”, manifesta forse un'altra isotopia che il lessema portava con sé, quella della velocità: senza che le definizioni dizionariali la nominino, tuttavia il contesto discorsivo sembra implicarla. Alla voce "urgente" leggiamo invece: "1) che deve essere fatto, risolto al più presto; 2) (ant, lett.) che preme, che incalza, che incombe". Questo secondo significato, che il dizionario giudica antico e letterario, pare manifestarsi in questo discorso. Alla voce "fronteggiare" leggiamo infatti (1): "contrastare validamente; tenere testa, far fronte (ad un attacco, a un assalto).

${ }^{11}$ Fra i significati dizionariali del termine: "agg. Fatto di nascosto, segretamente per lo più perché vietato dalla legge o da un autorità". Va inoltre notato che il dizionario registra "clandestino" come aggettivo, mentre nel testo esso ha funzione di sostantivo (come del resto, spesso, nei mezzi di comunicazione di massa e nel discorso politico italiano): il passaggio del termine a sostantivo da aggettivo si configura come una nominalizzazione implicitamente concettuale (si tratta di una delle metafore grammaticali individuate da M. A. K. Halliday): da carattere annesso a dei fenomeni per la loro segretezza od opposizione al potere politico, esso passa a indicare degli individui. La sostantivazione dell'aggettivo ha inoltre prodotto il termine "clandestinità", ma con questo passaggio la condizione che esso indica da stato temporaneo e modificabile diviene una condizione perpetua, tanto da definire degli individui. Cfr. Grande Dizionario Garzanti della lingua italiana 1987.

12 Il lessema "nomade" è definito dal dizionario come "caratterizzato dal nomadismo", a sua volta definito come "regime di vita proprio di popoli o tribù che non vivono in 


\section{Ocula $\mathbf{a}^{13}$}

Architettura e politica: un incrocio di sguardi | Pierluigi Cervelli, Politiche della sicurezza e forme del controllo. Una prospettiva semiotica.

Pur adottando modalità enunciative oggettivanti, simili a quelle tipiche del discorso scientifico, il testo non mette in scena procedure di dimostrazione delle affermazioni o di validazione di ipotesi tramite la costruzione di referenti interni.

Questo è evidente anche nelle "Linee guida per l'attuazione delle ordinanze del Presidente del Consiglio dei ministri del 30 maggio 2008, $\mathrm{n}^{\circ}$ $3676,3677,3678$, concernenti insediamenti di comunità nomadi nelle regioni Campania, Lazio e Lombardia" in cui il riferimento che potrebbe convalidare le affermazioni è al senso comune: «la dichiarazione dello stato di emergenza per le criticità relative ad insediamenti di comunità nomadi (...) è stata determinata, com'è noto, dalla grave situazione di degrado igienico, sanitario e socio-ambientale che si registra negli insediamenti abusivi ed anche in quelli autorizzati». Tramite l'inserimento dell'affermazione "com'è noto" il testo pone come suo presupposto il sapere senza fonti di una massa anonima. Piuttosto che essere però indebolito da questa indeterminatezza esso costruisce attorno ad essa la propria proposta di verità e la propria forza argomentativa.

Come notato da Sara Saleri ${ }^{14}$ le caratteristiche temporali di questa emergenza, il suo inizio e la sua durata, restano indeterminate e allo stesso modo restano indefiniti gli attori dell'emergenza: «Si tratta in prevalenza di persone senza fissa dimora che si spostano sul territorio creando insediamenti abusivi temporanei» 15 . Anche dal punto di vista spaziale l'unica indicazione che proviene dal testo è quella di una indefinibilità della localizzazione del fenomeno considerato.

Quello che in questi testi si costruisce, di cui il titolo rappresenta una sintesi, è la "finalità senza fine" tipica della articolazione tensiva della paura $^{16}$, che permette al linguaggio della sicurezza di creare i suoi attori senza volto e di legittimare le forme di controllo che i provvedimenti prevedono.

maniera stabile su un territorio, ma si spostano entro aree più o meno vaste a seconda degli andamenti stagionali e climatici", citando per esemplificare proprio "il nomadismo degli zingari”. Cfr, Grande Dizionario Garzanti 1987.

13 Occorre inoltre notare una contraddizione: nel testo queste persone vengono definite "nomadi" e "popolazioni senza territorio", ma si richiede loro, per l'ammissione nei nuovi campi-sosta, la dimostrazione di "soggiorno continuativo" sul territorio cittadino. Questo pone un ulteriore problema, di cui non possiamo occuparci in questa sede per ragioni di spazio, che è quello della difficoltà di definizione dell'alterità, e della distorsione che "l'altro" subisce quando viene introdotto dall'extrasemiotico nel semiotico (cfr. Lotman 1985).

14 Cfr. Saleri (2010).

15 Linee guida per l'attuazione delle ordinanze del presidente del consiglio dei ministri del 30 maggio 2008, $\mathrm{n}^{\circ} 3676,3677,3678$, concernenti insediamenti di comunità nomadi nelle regioni Campania, Lazio e Lombardia" (pag. 4).

${ }^{16}$ Cfr Lotman 2008 e Pezzini 1998. 


\section{Ocula ${ }^{13}$}

Architettura e politica: un incrocio di sguardi | Pierluigi Cervelli, Politiche della sicurezza e forme del controllo. Una prospettiva semiotica.

\section{Forme del controllo: spazio visibile e confini mobili}

3.1 La costruzione del sapere: individuazione e individualizzazione degli attori

Le modalità di opposizione al pericolo avranno allora a che fare con la costruzione di un sapere e di una nuova visibilità attraverso il controllo del movimento nello spazio e dei tempi in cui esso si effettua, oltre all'identificazione dei soggetti in movimento.

Obiettivo primario sarà identificare e censire la popolazione fluttuante: la "precisa individuazione dell'ubicazione degli insediamenti autorizzati e abusivi. Accanto al complessivo monitoraggio della composizione, specie numerica, degli insediamenti, occorre poi concretizzare la rilevazione delle presenze, eventualmente a mezzo di un apposito foglio notizie" 17 garantendo la "necessaria identificazione". L'assicurazione di "mezzi certi di identificazione", avverrà attraverso: «b) monitoraggio dei campi autorizzati in cui sono presenti comunità nomadi ed individuazione degli insediamenti abusivi; c) identificazione e censimento delle persone, anche minori di età, $\mathrm{e}$ dei nuclei familiari presenti nei luoghi di cui al punto b), attraverso rilievi segnaletici c) (...) individuazione di altri siti (...)»18. Questa norma è stata particolarmente contestata perché ha previsto che fossero prese le impronte digitali a tutti gli abitanti dei campi, compresi i bambini che avessero compiuto i due anni di età: di fatto si è trattato dell'estensione di una pratica di polizia a persone non accusate di reato, che ha caratterizzato su base etnica il censimento svolto. Il sapere costruito servirà a spostare la popolazione cambiandone le modalità abitative attraverso «la costruzione di quattro villaggi della solidarietà in aree attrezzate in grado di ospitare circa 1000 persone - ciascuno da realizzare su aree comunali o demaniali disciplinati da specifici regolamenti di gestione»19. Nei nuovi siti si provvederà alla costruzione di un tipo di campo-sosta molto diverso dai precedenti, a partire dalla suddivisione dello spazio individuale. Negli insediamenti autorizzati la disposizione prossemica era basata sulle relazioni parentali: prevedeva come unità di riferimento la famiglia allargata, i cui membri si disponevano in maniera contigua e la separazione spaziale delle famiglie diverse e soprattutto di quelle in conflitto. I campi non autorizzati seguivano la stessa logica, essendo composti però solo da una o due famiglie.

I nuovi campi saranno invece divisi in unità elementari tutte uguali, disposte generalmente a scacchiera, e ad ogni famiglia verrà assegnata una piazzola individuale. Per cui l'oggetto che sarà reso visibile all'interno del campo avrà una grana molto più fine rispetto a quelli citati nei testi precedenti: stavolta non si fa riferimento ad una massa indifferenziata, in movimento e proliferante, ma piuttosto ad un elemento minimo su cui si

17 "Linee guida per l'attuazione delle ordinanze del presidente del consiglio dei ministri del 30 maggio 2008, $\mathrm{n}^{\circ} 3676,3677,3678$, concernenti insediamenti di comunità nomadi nelle regioni Campania, Lazio e Lombardia" (pag. 2).

18 Ordinanza del Presidente del Consiglio dei ministri, 30 maggio 2008, pag.1.

19 Patto per Roma sicura 2007, pag. 3. "Villaggi della solidarietà" è il nome che è stato dato a Roma ai nuovi campi sosta.

7 | www.ocula.it | agosto 2012 


\section{Ocula ${ }^{13}$}

Architettura e politica: un incrocio di sguardi | Pierluigi Cervelli, Politiche della sicurezza e forme del controllo. Una prospettiva semiotica.

applicheranno le disposizioni, e che avrà la taglia della famiglia, e specificamente del singolo nucleo familiare.

La massa che le disposizioni urgenti indicavano come creatrice delle "criticità", verrà così scomposta e articolata, tramite la suddivisione spaziale delle aree di sosta, in tante parti tutte uguali ed il controllo si effettuerà su queste parti singolarizzate. Si tratta del primo passo per la costruzione di una competenza cognitiva di un attante "di controllo", "sovrapposto" agli attori individuali presenti nel campo.

\subsection{L'articolazione spaziale: il confine mobile}

Ma questa articolazione in unità minime, cosa permetterà di controllare? Essenzialmente la mobilità dal "campo" allo spazio urbano: tutte le presenze nel campo e gli ingressi dei presenti saranno registrati e verificati così come tutti gli ingressi degli ospiti saranno registrati e controllati20: "la vigilanza del villaggio è assicurata da un Presidio istituito anche per il controllo degli accessi”"21, (che potrà anche essere composto da vigilanza privata). Il presidio di vigilanza "cura la compilazione del registro delle presenze degli abitanti del villaggio e ne verifica l'identità all'ingresso" e "cura la compilazione di un registro per l'identificazione dei visitatori occasionali, previo accertamento del consenso del nucleo familiare di riferimento". Inoltre: "Potranno anche essere previsti l'installazione e l'utilizzo di strumenti tecnologici atti a rafforzare i controlli e la sicurezza del villaggio" nonché nel "perimetro esterno si prevedono altresì forme di vigilanza delle forze dell'Ordine" 22 .

L'art. 11, che disciplina le visite, recita al comma 1: "I parenti, gli amici, e i conoscenti degli ospiti possono accedere liberamente al campo per recarsi dall'ospite che intendono visitare, facendosi identificare all'ingresso dal gestore sociale" (corsivi miei). Il nuovo campo non è dunque un posto da cui si entra o si esce senza motivo: per entrare si deve avere qualcuno da cui andare (la visita dovrà essere connessa a una delle unità minime che compongono il campo) e per uscire bisognerà dare comunicazione: l'assenza ingiustificata comporterà infatti l'espulsione dal campo stesso. In questo modo, oltre al controllo dei piccoli movimenti individuali, sarà controllato anche il movimento più lungo, quello del viaggio ${ }^{23}$.

Altro aspetto importante, il confine del campo potrà restringersi o allargarsi "per comprovati motivi di sicurezza": il comma 4 dell'art 11 recita: "il comitato di gestione può temporaneamente sospendere l'afflusso delle aree di sosta avvisando tempestivamente gli ospiti" e l'art. 13 specifica che "per prevenire ed eliminare gravi pericoli che minacciano l'incolumità

${ }^{20}$ Uno specifico documento di riconoscimento, "una tessera munita di fotografia recante i dati anagrafici (...) e valida solo ai fini dell'accesso al villaggio”, verrà a questo scopo assegnato ad ogni componente di ogni nucleo familiare.

${ }^{21}$ Articolo 2.4 (Vigilanza) del regolamento dei "villaggi della solidarietà" di Roma.

22 ibidem.

${ }^{23}$ L'autorizzazione è revocata all'interno nucleo familiare (art. 12 del regolamento del Comune di Milano) qualora vi sia "abbandono della struttura assegnata per un periodo superiore ad un mese salvo espressa e preventiva autorizzazione del comitato. 


\section{Ocula ${ }^{13}$}

Architettura e politica: un incrocio di sguardi | Pierluigi Cervelli, Politiche della sicurezza e forme del controllo. Una prospettiva semiotica.

pubblica e la sicurezza urbana le aree di sosta possono essere chiuse in ogni tempo dal comune per sopravvenuti motivi di pubblico interesse"24. La relazione di inglobamento che il campo istituisce non è dunque stabilita una volta per tutte ma articolabile per intensità: il confine dilata o restringe continuamente la sua porosità, rendendosi cioè mobile come erano mobili le persone di cui esso circoscrive ora lo spazio. La logica del limite poroso non è inoltre provvisoria o emergenziale: tutto il campo è sottoposto nella sua interezza alla dinamica che investe normalmente il suo limite25.

Quella che avverrà ai limiti del campo non sarà dunque una semplice registrazione degli ingressi e delle uscite ma una regolazione degli accessi e un controllo dei confini: se gli spazi individuali sono ascrivibili (seppure temporaneamente e con dei limiti) agli attori che li abitano, le famiglie, i confini fra l'interno e l'esterno del campo sembrano invece ascritti al potere di un attante sovra individuale, che non è riconducibile alla collettività degli attori presenti nel campo e che ha la possibilità di controllare, restringere 0 espandere gli accessi e le uscite. I confini dell'insieme costituiscono così uno spazio autonomo rispetto al campo: uno spazio semanticamente "non privato" e politico, che non coincide col limite dei confini delle unità individuali ma le ingloba secondo la logica di un potere esterno ad esse, definendo l'articolazione modale dei soggetti che le attraversano.

\subsection{L'articolazione del tempo: la provvisorietà strutturale}

L'ingresso all'interno dei campi sosta, che avverrà tramite una procedura di assegnazione di posti limitati, avrà come esito l'impossibilità di una sistemazione permanente: "L'ammissione al villaggio comporta la temporanea (corsivo mio) assegnazione di una struttura abitativa, anche prefabbricata o realizzata con tecniche di autocostruzione, ovvero di una piazzola di sosta per roulottes e moduli abitativi” ${ }^{26}$. Se si entra nel villaggio si è tendenzialmente tenuti a starvi sempre ma sarà comunque necessario uscirne, prima o poi: nel Lazio le autorizzazioni alla sosta avranno validità biennale, prorogabile per due anni e poi ulteriormente per altri due, ma questa possibilità è prevista solo come possibilità per il dipartimento delle Politiche Sociali, "per completare i percorsi di integrazione socio-educativa";

24 Mi riferisco agli art. 11 e 13 del regolamento emesso riguardo al Comune di Milano. Provvedimenti analoghi sono previsti all'art. 5 del regolamento che riguarda la regione Lazio.

25 La chiusura all'esterno (temporanea) o quella permanente possono essere decise al di là delle volontà degli abitanti del campo (che tuttavia pagano un affitto per la sosta) e in qualunque momento: questa possibilità manifesta il tratto di "provvisorietà strutturale" rilevato per quanto riguarda l'articolazione temporale della sosta.

${ }^{26}$ L'articolo 1 del regolamento previsto dal Comune di Milano precisa che esso "disciplina le aree destinate alla sosta transitoria dei nomadi" e che la permanenza all'interno dei campi è di un anno, prorogabile, senza fornire altre indicazioni in merito. Gli articoli 3.4 (validità delle autorizzazioni) e 3.5 (proroga delle autorizzazioni) del regolamento per la gestione dei villaggi nella regione Lazio pongono dei limiti precisi: due anni prorogabili per altri due, più eventualmente altri due anni. È possibile presumere dunque che dopo questo periodo sia prevista l'espulsione dai campi. 


\section{Ocula ${ }^{13}$}

Architettura e politica: un incrocio di sguardi | Pierluigi Cervelli, Politiche della sicurezza e forme del controllo. Una prospettiva semiotica.

nei campi sosta all'interno del comune di Milano la sosta è invece prevista per un solo anno. La temporalità specifica del nuovo campo-sosta introduce dunque una provvisorietà strutturale nella permanenza dei soggetti autorizzati: dopo un certo periodo di tempo si dovrà comunque lasciare il campo. La provvisorietà strutturale della permanenza rovescia così la temporalità abitativa degli insediamenti dispersi, che erano mobili per permettere, nonostante la frequenza degli sgomberi, di ritornare sempre nello stesso posto27. I "nuovi" nomadi vivranno infatti una provvisorietà del tutto differente rispetto al passato: alla mobilità precaria nello spazio e alla continuità del tempo che caratterizzava i precedenti insediamenti abusivi si sostituiscono ora una fissità nello spazio (attraverso la creazione di una spazialità individuale e il controllo del movimento) e una mobilità nel tempo, attraverso l'introduzione dei limiti temporali e delle proroghe, concedibili «sulla base dei complessivi comportamenti tenuti dal nucleo familiare (...) e previa verifica della puntualità di pagamento delle utenze di relativa spettanza» ${ }^{28}$. La revocabilità e la provvisorietà sono i tratti su cui si costruirà un tempo mobile, la cui durata potrà essere provvisoriamente allungata $\mathrm{e}$ sarà comunque interrotta, pur rispettando le regole che disciplinano $\mathrm{i}$ comportamenti individuali e familiari. In questo modo però i provvedimenti sulla sicurezza producono di fatto i nomadi ai cui comportamenti abitativi hanno imputato la loro stessa necessità.

Quello che questa modalità di soggiorno costruisce è infatti una costrizione al nomadismo: se quello che causa allarme era un nomadismo individuale teso alla permanenza temporale nel territorio, questo che il regolamento disegna è un nomadismo di massa, in cui la permanenza sarà solo una sospensione del movimento. I regolamenti svelano così il loro carattere di "testi programmatori": non mirano però alla produzione di oggetti, come nel caso analizzato da A. J. Greimas ${ }^{29}$, ma alla produzione di forme di vita.

\section{Conclusioni}

I dispositivi di sicurezza mirano a produrre frontiere anelastiche e indeformabili3o, attraverso cui controllare la circolazione degli individui. Ma in questo modo i provvedimenti disegnano un nuovo spazio politico: quello

27 Emblematico il caso del campo abitato da circa 50 sinti italiani che lo scorso anno a Milano è stato sgomberato 52 volte in otto mesi, dunque ogni quattro giorni circa, e per 52 volte è stato ricostituito nello stesso posto. Cfr. Corriere della sera, 1/o8/2009, "Il campo nomadi sgomberato 52 volte", articolo di Andrea Senesi. Interessante anche notare che, nonostante quanto si affermi nell'articolo, il box aggiuntivo che lo affianca definisce rom e sinti "I nomadi". Va inoltre ricordato che nel campo di Casilino 900, recentemente sgomberato a Roma, alcune famiglie abitavano con continuità da 20 e anche 30 anni.

28 Cfr. l'art. 3.6 del "Regolamento per la gestione dei villaggi attrezzati per le comunità nomadi nella Regione Lazio".

29 Cfr. Greimas 1983.

30 Sul concetto di frontiera anelastica, e in generale sulla relazione fra architettura e controllo territoriale nel contesto del conflitto israelo-palestinese cfr. Weizman 2009. 


\section{Ocula ${ }^{13}$}

Architettura e politica: un incrocio di sguardi | Pierluigi Cervelli, Politiche della sicurezza e forme del controllo. Una prospettiva semiotica.

di un territorio in cui circolano in maniera imprevedibile o comunque fortemente mobile degli elementi negativi da eliminare. Il territorio in cui la negatività si muove senza sosta dovrà essere popolato da una serie indefinita di elementi di controllo, potenzialmente in grado di identificarla ovunque. $\grave{E}$ proprio l'immagine dello spazio sottesa a questo tipo di topologia mobile che pare mutata profondamente rispetto al territorio delle discipline ${ }^{31}$ descritto in profondità da M. Foucault: lontano dall'essere un insieme fisso di elementi esso è ora concepito come un campo attraversato da flussi di circolazione. È proprio la circolazione del pericolo, che all'interno di esso si presuppone in costante spostamento ed evoluzione, a dover produrre una geografia mobile del controllo. Ma questa geografia, estesa a tutto lo spazio urbano, non riguarda più solo i rom ma potenzialmente tutti i cittadini. Sperimentata sulla popolazione marginale delinea una politica dello spazio urbano: basti pensare al modo in cui la messa in sicurezza dello spazio privato si correla alle pratiche di controllo, riduzione e gestione dello spazio pubblico messe in atto in alcuni nuovi quartieri residenziali della città di Roma e più in generale alle politiche di gestione dello spazio pubblico, anche alla luce delle recenti ordinanze comunali emesse in Italia fra il 2008 ed il 2009, tese a disciplinare i comportamenti degli utilizzatori32.

Una riflessione sulle interazioni fra architettura e politica sembra così condurci, attraverso la semiotica dello spazio, al cuore di quella che Greimas (1983) chiamava, riferendosi non casualmente a Michel Foucault, una semiotica del potere.

\section{Bibliografia}

Cervelli P.

2008 "Vuoti, Stratificazioni, Migrazioni. Programmazioni urbanistiche e forme dell'abitare a Roma" in: Lexia, n.1, a cura di Leone M., "La città come testo. Scritture e riscritture urbane"

Fabbri, P., Marcarino, A.

1985 "Il discorso politico" in: Carte Semiotiche, n. 1, 1985, Firenze, La Casa Usher, Firenze.

Foucault, M.

2004 Securité, territoire, population. Cours au Collège de France (1977-1978), Paris, Seuil/Gallimard (tr. it., Sicurezza, territorio, popolazione. Corso al Collège de France (1977-1978), Milano, Feltrinelli, 2007).

Greimas, A. J., Courtés, J.

1979-2007 Sémiotique. Dictionnaire raisonné de la théorie du langage, Paris, Hachette (tr. it., Semiotica. Dizionario ragionato della teoria del linguaggio, a cura di Fabbri, P., Milano, Bruno Mondadori, 2007).

${ }^{31}$ Cfr. Foucault (1978) in particolare sul passaggio dalla logica degli spazi disciplinari a quella della sicurezza della popolazione.

32 Elena Lorenzetto ne ha contate ben 788, emesse in un solo anno fra il 2008 ed il 2009. Cfr. Lorenzetto 2010.

11 | www.ocula.it | agosto 2012 


\section{Ocula ${ }^{13}$}

Architettura e politica: un incrocio di sguardi | Pierluigi Cervelli, Politiche della sicurezza e forme del controllo Una prospettiva semiotica.

Greimas, A. J.

1983 Du sens II, Paris, Seuil; (tr. it., Del senso 2, Milano, Bompiani, 1985).

Hammad, M.

2003 Leggere lo spazio, comprendere l'architettura, Roma, Meltemi.

Weizman, E.

2007 Hollow Land, London, Verso (tr. it., Architettura dell'occupazione, Bruno Mondadori, Milano, 2009).

Landowski, E.

1989 La société réfléchie, Paris, Editions du Seuil (tr. it., La società riflessa, Roma, Meltemi, 2001).

Lorenzetto, E.

2010 "Ordinare gli spazi. Rassicurare la città. Un'analisi socio semiotica delle politiche locali sulla sicurezza urbana”, in: $E / C$, rivista on-line dell'Associazione Italiana di Studi Semiotici.

Lotman, J. M.

1998 “Ochota za ved'mami. Semiotika stracha”, in: Trudy po znakovym sistemam, vol. 126 (tr. it., "La caccia alle streghe. Semiotica della paura", in: $E / C$, rivista on-line dell'Associazione Italiana di Studi Semiotici, 2008).

Montanari F.

2010 "Introduzione. Le forme, vecchie e nuove, del discorso politico", in: Montanari F., a cura, Politica 2.o, Roma, Carocci.

Pezzini, I.

1998 Le passioni del lettore, Milano, Bompiani.

Piasere, L.

1999 Un mondo di mondi, Napoli, L'ancora.

Saleri, S.

2010 "Emergenze sicurezza: un discorso istituzionale sull'alterità", in: $E / C$, rivista on-line dell'Associazione Italiana di Studi Semiotici. 